\title{
APTES Modification of Molybdenum Disulfide to Improve the Corrosion Resistance of Waterborne Epoxy Coating
}

\author{
Yang Liu ${ }^{1,2}$, Shihong Zhang ${ }^{2, *}$, Yi He ${ }^{1,2, *}$, Chunlin Chen ${ }^{1,2}$, Chen Zhang ${ }^{1,2}$, Peng Xie ${ }^{1,2}$, Fei Zhong ${ }^{1}$, \\ Hongjie Li ${ }^{1,2}$, Jingyu Chen ${ }^{3}$ and Zhenyu $\mathrm{Li}^{3}$ \\ 1 State Key Lab of Oil and Gas Reservoir Geology and Exploitation, Southwest Petroleum University, Rd. 8, \\ Xindu District, Chengdu 610500, China; ly13032827010@163.com (Y.L.); 13541396226@163.com (C.C.); \\ xu_jiangyu@126.com (C.Z.); xp13438370660@126.com (P.X.); 13880298240@163.com (F.Z.); \\ 202011000056@stu.swpu.edu.cn (H.L.) \\ 2 College of Chemistry and Chemical Engineering, Southwest Petroleum University, 8 Xindu Avenue, \\ Xindu District, Chengdu 610500, China \\ 3 Chengdu Evermaterials Tec Co, Chengdu 610500, China; jingyu.chen@deakin.edu.au (J.C.); \\ zhenyu.li@deakin.edu.au (Z.L.) \\ * Correspondence: hong73787@163.com (S.Z.); chemheyi@swpu.edu.cn (Y.H.); Tel.: +86-028-8303-7306 (Y.H.)
}

Citation: Liu, Y.; Zhang, S.; He, Y.; Chen, C.; Zhang, C.; Xie, P.; Zhong, F.; Li, H.; Chen, J.; Li, Z. APTES Modification of Molybdenum Disulfide to Improve the Corrosion Resistance of Waterborne Epoxy Coating. Coatings 2021, 11, 178. https://doi.org/10.3390/ coatings11020178

Academic Editor: Cecilia Bertuli

Received: 7 January 2021

Accepted: 27 January 2021

Published: 2 February 2021

Publisher's Note: MDPI stays neutral with regard to jurisdictional claims in published maps and institutional affiliations.

Copyright: (c) 2021 by the authors. Licensee MDPI, Basel, Switzerland. This article is an open access article distributed under the terms and conditions of the Creative Commons Attribution (CC BY) license (https:/ / creativecommons.org/licenses/by/ $4.0 /)$.

\begin{abstract}
MoS}_{2}$ has been regarded as a promising addition for the preparation of epoxy-based coatings with high anticorrosion ability. However, its dispersion and compatibility remain significant challenges. In the present work, an organic thin layer was well coated on lamellar molybdenum disulfide $\left(\mathrm{MoS}_{2}\right)$ via a simple modification of 3-aminopropyltriethoxysilane (APTES). The modification of hydrolyzing APTES on lamellar $\mathrm{MoS}_{2}$ effectively improved the dispersity of $\mathrm{MoS}_{2}$ in water-borne epoxy (WEP) and successfully enhanced the compatibility and crosslinking density of $\mathrm{MoS}_{2}$ with WEP. The influence of introducing $\mathrm{MoS}_{2}$-APTES into WEP coating on anticorrosion property for N80 steel was tested by electrochemical impedance spectroscopy (EIS), potentiodynamic polarization and salt spray test. The results exhibited that the $|\mathrm{Z}|_{0.01 \mathrm{~Hz}}$ value of $\mathrm{MoS}_{2}$-APTES/WEP still reached $3.647 \times 10^{7} \Omega \cdot \mathrm{cm}^{2}$ even after the immersion time of 50 days in $3.5 \mathrm{wt} . \% \mathrm{NaCl}$ solution, showing an extraordinary performance of corrosion resistance. The enhanced anticorrosion performance of composite coating could be resulted from the apparently increased dispersibility and compatibility of $\mathrm{MoS}_{2}$ in WEP.
\end{abstract}

Keywords: molybdenum disulfide $\left(\mathrm{MoS}_{2}\right)$; 3-aminopropyltriethoxysilane; waterborne epoxy; anticorrosion

\section{Introduction}

Metal corrosion has become a worldwide problem causing enormous economic losses and many casualties [1,2]. Among the conventional anticorrosive strategies, organic coatings have generated tremendous attention from researchers and have been extensively used in industrial fields, mainly due to their superior physical barrier abilities and relatively low costs [3-5]. However, solvent-based coatings containing many volatile organic compounds (VOCs) will heavily damage the eco-environment and human health [6,7]. Waterborne epoxy coating (WEP), one of the environment-friendly coatings, has drawn an increasing amount of attention owing to its many virtues like low noxiousness and excellent chemical resistance [8-10]. Unfortunately, WEP coatings could not attain satisfactory results of protecting metal from corrosion due to the formation of micro-pores or micro-cracks during coating curing caused by solvent evaporation [11]. To solve this problem, some methods have been developed to reinforce the anticorrosive capability of the WEP coatings. Among these diverse methods, various fillers such as graphene and its derivates, $\mathrm{C}_{3} \mathrm{~N}_{4}$, and $\mathrm{h}-\mathrm{BN}$, etc., have been introduced into the waterborne epoxy, which is an effective way to reduce coating defects and promote the anticorrosion performance of WEP coatings [12]. 
To date, graphene, one of the two-dimensional (2D) materials, has already generated an increasing amount of interest among researchers in the area of anticorrosion, because of its superior impermeability to molecules, excellent chemical inertness, hydrophobicity, outstanding thermal and mechanical properties, etc. [13]. However, it is also well known that graphene cannot function as a long-term anticorrosion material, and it even accelerates metal corrosion once the coating is scratched [14]. This is a tremendous limit to graphene applied to anticorrosion. Even though previously relevant "self-healing" strategies can extend the life of graphene-based coatings, they cannot basically hinder the "corrosion-promotion activity". Consequently, it is particularly crucial to hinder the corrosion-promotion activity of graphene-based anticorrosion coatings or find low conductivity/insulation materials $[9,15]$.

$\mathrm{MoS}_{2}$, as a member of the transition metal dichalcogenides, has attracted a significant interest due to its 2D ultrathin atomic layer structure similar to that of graphene [16,17]. It is made up of one plane of hexagonally packed metal atoms sandwiched by two planes of chalcogenide atoms. The sandwich layers stack vertically and are loosely bonded via weak van der Waals forces $[18,19]$. The special lamellar structure of $\mathrm{MoS}_{2}$ endows it with excellent anticorrosion performance-like graphene [20]. Meanwhile, $\mathrm{MoS}_{2}$ possesses a high band gap in contrast to graphene, which has no influence on the electrical conductivity of the coatings when added with the $\mathrm{MoS}_{2}$ [21,22]. As a result, $\mathrm{MoS}_{2}$-based anticorrosion coatings will not cause "micro-galvanic corrosion" if the defects occur on the surface of the compounded coatings [23]. However, $\mathrm{MoS}_{2}$ has a tendency to agglomerate in the polymer matrix, because it possesses a large surface area and is short of functional groups. To improve its dispersibility and compatibility with the polymer matrix, the most effective methods to prepare high-performance composites is the surface modification of $\mathrm{MoS}_{2}$ including the non-covalent and covalent types [24]. For example, Wang et al. [25] found a non-covalently functionalized method to modify $\mathrm{MoS}_{2}$ with chitosan (CS), and the CS$\mathrm{MoS}_{2}$ could be homogeneously dispersed in tetrahydrofuran, which could greatly decrease hazards connected with EP nanocomposites. Zhou et al. [26] devised an approach of covalent functionalization to graft $\mathrm{MoS}_{2}$ nanosheets with 3-mercaptopropyl trimethoxysilane and the functionalized $\mathrm{MoS}_{2}$ could be well dispersed in PVA without obvious aggregation.

Moreover, it is generally acknowledged that organic silane coupling agents could be used to modify materials. In this regard, Sepideh et al. [27] used (3-aminopropyl) triethoxysilane (APTES)-modified graphene oxide (GO) as a nanofiller to add into the epoxy coating, and the results showed that corrosion protection performance greatly improved because of the excellent interfacial interaction of GO in coating through silane modification. Sepideh et al. [28] reported 3-aminopropyl triethoxysilane (APTES) and 3glysidyloxypropyl trimethoxysilane (GPTMS) as silane agents to modify GO sheets, which were introduced to epoxy coatings. The results exposed the incorporation of APTES-GO including amine end-groups, which better increased the corrosion resistance of epoxy coating. As we all know, APTES, a kind of typical silane coupling agent, could hydrolyze and graft to the surface of fillers, and its amine-end groups could improve the crosslinking density of epoxy resins.

In this study, $\mathrm{MoS}_{2}$ was functionalized with APTES in order to develop its dispersion and interfacial interactions with waterborne epoxy. Then, the hybrids $\mathrm{MoS}_{2}$-APTES were introduced into waterborne epoxy resin coatings to strengthen the anticorrosion performance. To the best of our knowledge, there is no study reporting the anticorrosion performance of APTES-modified $\mathrm{MoS}_{2}$ in waterborne epoxy. Finally, the corrosion resistance of the composite coatings was investigated by electrochemical impedance spectra (EIS), potentiodynamic polarization and salt spray test.

\section{Experiment}

\subsection{Materials}

Molybdenum disulfide $\left(\mathrm{MoS}_{2}, 99.9 \%\right)$ was obtained from McLean Biotech (Shanghai, China), and 3-aminopropyltriethoxysilane (APTES, 99\%) and ethanol ( $\geq 99.7 \%)$ were 
purchased from Kelong Chemical Reagent Factory (Chengdu, China). Waterborne epoxy $(\mathrm{BC} 2060 \mathrm{H})$ and curing agent (BC916) provided by LianGu New Material Technology Co., Ltd. (Guangdong, China) were used to prepare the coatings. Distilled water was produced by the Milli-Q system (Millipore, NY, USA). All the reagents used in the experiment were directly utilized without other purification steps.

\subsection{Preparation of $\mathrm{MoS}_{2}$-APTES}

APTES was employed to modify $\mathrm{MoS}_{2}$ as follows: $0.3 \mathrm{~g} \mathrm{MoS}_{2}$ was dispersed in $150 \mathrm{~mL}$ ethanol including $3 \mathrm{~g}$ APTES by sonification for $40 \mathrm{~min}$, and then $5 \mathrm{~mL}$ of distilled water was dropped in the prepared suspension under sonification. The suspension was magnetically stirred at $500 \mathrm{rpm}$ for $24 \mathrm{~h}$ at $80^{\circ} \mathrm{C}$. Then, modified $\mathrm{MoS}_{2}$ was centrifuged, washed several times with pure water and ethanol to insure the complete removal of unreacted APTES. In the end, the acquired products were dried by freeze-drying. The schematic of the synthesis procedure of $\mathrm{MoS}_{2}$-APTES was displayed in Figure 1.

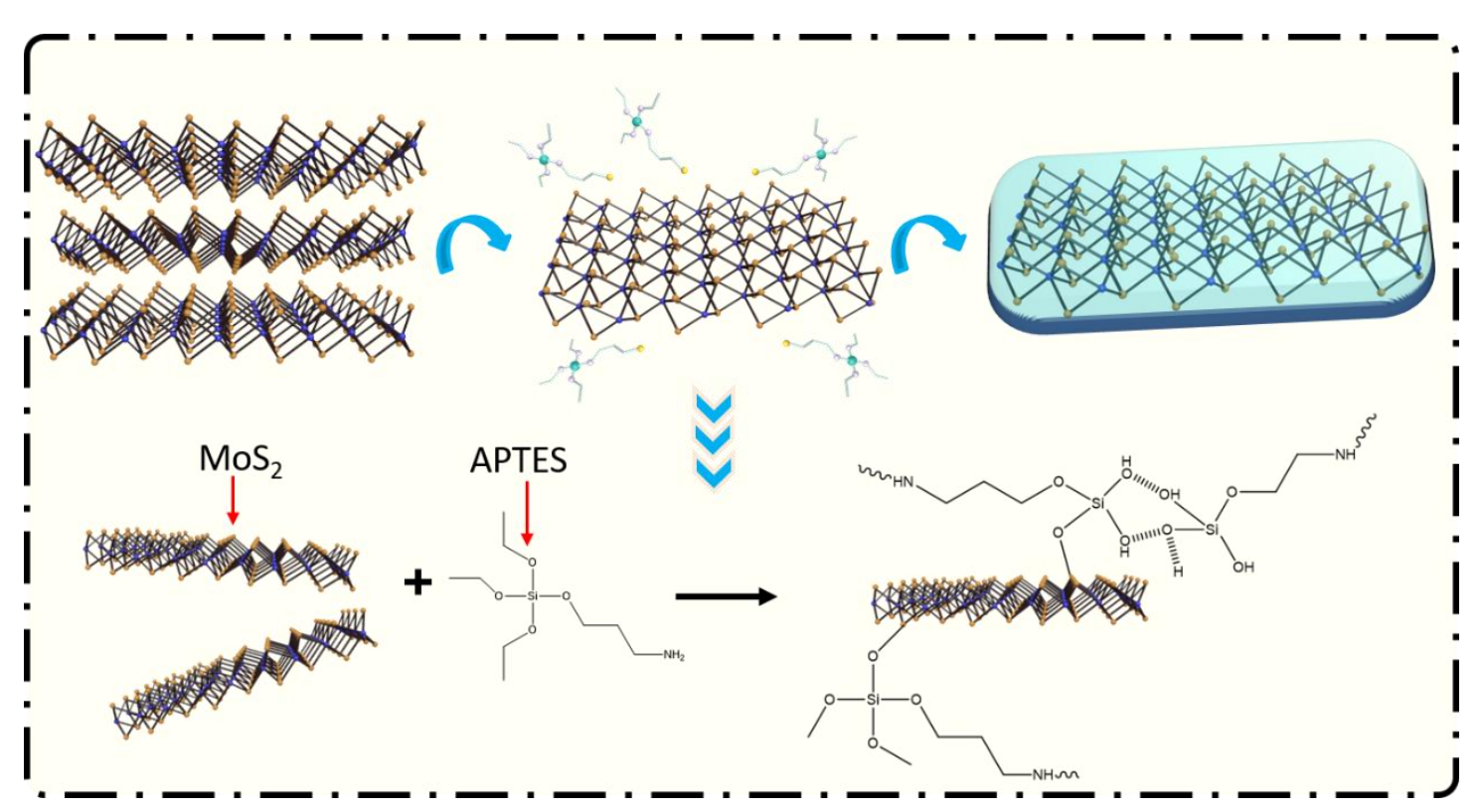

Figure 1. Schematic of the synthesis procedure of $\mathrm{MoS}_{2}$-3-aminopropyltriethoxysilane (APTES) via APTES hydrolysis.

\subsection{Fabrication of $\mathrm{MoS}_{2}$-APTES Composite Coating}

Firstly, a certain amount of $\mathrm{MoS}_{2}(0.3 \mathrm{wt} . \%)$ was dispersed in pure water, stirred and ultrasonicated for $5 \mathrm{~min}$ to obtain a homogeneous solution. Subsequently, the $\mathrm{MoS}_{2}$ APTES suspension was added into waterborne epoxy resin containing curing agent and mechanically stirring for $10 \mathrm{~min}$ to produce a homogeneous mixture. Finally, the mixture was sprayed on the prepared N80 steel substrates degreased by ethanol and acetone. The composite coating was placed at $25^{\circ} \mathrm{C}$ for 7 days to promote curing. The average thickness of the coating was measured by the coating thickness gauge (QNIX 4500, Qnix, Bonn, Germany), which was about $40.0 \pm 5 \mu \mathrm{m}$. Similarly, The WEP and $\mathrm{MoS}_{2} / \mathrm{WEP}$ were also prepared via the same method.

\subsection{Characterization}

Fourier transform infrared (FT-IR, WQF-520, Rui Li, Beijing, China) was used to initially characterize the chemical structure of the composite in the range of $500-4000 \mathrm{~cm}^{-1}$. To investigate the crystal structure of materials, X-ray diffraction (XRD, Bruker D8 ADVANCE A25X, Beijing, China) was carried out using copper Ka radiation source at a scan rate of $1^{\circ} \cdot \mathrm{s}^{-1}$ from $5^{\circ}$ to $80^{\circ}$ and X-ray diffraction measurement was in Bragg-Brentano geometry. The morphology of $\mathrm{MoS}_{2}$-APTES was characterized by transmission electron microscopy 
(TEM, JEM-2100F, Japan Electron Optics Laboratory Co, Tokyo, Japan). X-ray photoelectron spectrometry (XPS, XSAM800, Al K $\alpha$ ray, KRATOS, Manchester, UK) was employed to explore the surface chemistry. Thermogravimetric analysis (TGA) was conducted by TGA (SDTA851, METTLER, Zurich, Switzerland) on the condition that $\mathrm{N}_{2}$ atmosphere flowed over the open crucibles and a heating temperature increased from $40-800{ }^{\circ} \mathrm{C}$ with a rate of $10^{\circ} \mathrm{C} / \mathrm{min}$. In order to study the dispersion ability of additions in WEP coatings, scanning electron microscopy (SEM, JSM-6700, JEOL, Tokyo, Japan) was utilized to survey the fracture surface of coatings after breaking the coatings in liquid nitrogen.

\subsection{Corrosion Measurements}

\subsubsection{Electrochemical Test}

The corrosion protection ability of the neat WEP, $\mathrm{MoS}_{2} / \mathrm{WEP}, \mathrm{MoS}_{2}$-APTES/WEP was investigated by electrochemical impedance spectra (EIS) measurements which were from the CorrTest CS350 in $3.5 \mathrm{wt} . \% \mathrm{NaCl}$ solution. Three traditional electrode systems including a working electrode (specimen coatings), reference electrode (saturated calomel electrode) and counter electrode (foil electrode) were applied in the measurement process. During the EIS test, the parameter of the frequency range was $10^{-2} \sim 10^{5} \mathrm{~Hz}$ and the open circuit potential (OCP) always remains stable. In addition, the test data were fitted and evaluated by the ZsimpWin software. Additionally, the potentiodynamic polarization test was employed to further study the anticorrosive performance of the composite coating, which was conducted with a scan rate of $1 \mathrm{mV} \cdot \mathrm{s}^{-1}$ from $-200 \mathrm{mV}$ (the cathodic direction) to $+200 \mathrm{mV}$ the anodic (direction).

\subsubsection{Salt Spray Test}

The long-term anticorrosion performance was estimated by conducting salt spray testing. According to the ASTM B117 standard, samples with a $4 \mathrm{~cm}$ scratch were exposed to $5 \mathrm{wt} . \% \mathrm{NaCl}$ salt spray.

\section{Results and Discussion}

\subsection{Characterization}

To confirm the successful modification of APTES on $\mathrm{MoS}_{2}$, FTIR spectroscopy of $\mathrm{MoS}_{2}$, $\mathrm{MoS}_{2}$-APTES was displayed in Figure 2a. From the figure, there is no obvious absorption peak in the spectrum of original $\mathrm{MoS}_{2}$, because of the lack of functional groups on the surface of $\mathrm{MoS}_{2}$ [29]. In the case of $\mathrm{MoS}_{2}$-APTES, some peaks at 2917 and $2850 \mathrm{~cm}^{-1}$ are associated with the symmetric and asymmetric stretching of $\mathrm{C}-\mathrm{H}$ [27]. Moreover, the broad absorbance between 3436 and $3308 \mathrm{~cm}^{-1}$ could be related to $\mathrm{O}-\mathrm{H}$ stretching of water and $\mathrm{N}-\mathrm{H}$ stretching vibrations. These prove the resultful modification of $\mathrm{MoS}_{2}$ with APTES. Furthermore, new bands at 1624, 1117, 1042, 912, 755, $\mathrm{cm}^{-1}$ are connected with the deformation (scissoring) of $\mathrm{N}-\mathrm{H}$, perpendicular $\mathrm{Si}-\mathrm{O}$ stretching vibrations, in-plane $\mathrm{Si}-\mathrm{O}$ stretching, $\mathrm{O}-\mathrm{H}$ deformation of hydroxyl groups and perpendicular $\mathrm{Si}-\mathrm{O}$ stretching vibrations, respectively [30]. All the above indicate that APTES has been successfully modified to the surface of $\mathrm{MoS}_{2}$.

XRD spectra were employed to investigate the crystalline structure of $\mathrm{MoS}_{2}$ and $\mathrm{MoS}_{2}$ APTES. As shown in Figure 2b, the crystalline diffraction peaks of $\mathrm{MoS}_{2}$ appear at $14.36^{\circ}$, $32.88^{\circ}$ and $58.02^{\circ}$, which correspond to (0:0:2), (1:0:0) and (1:1:0) planes, respectively [31]. After modification with APTES, the hybrids exhibit no peak shift in contrast to $\mathrm{MoS}_{2}$, which manifests that there is no influence on the crystalline phrase of $\mathrm{MoS}_{2}$ via surface modification. Moreover, it is clear that the intensity of the (0:0:2) diffraction peaks of the $\mathrm{MoS}_{2}$-APTES hybrids obviously decreased compared to that of $\mathrm{MoS}_{2}$, which can probably be attributed to the thin layer of APTES hindering the aggregation of $\mathrm{MoS}_{2}$. Overall, these results demonstrate that APTES is successfully grown on the surface of $\mathrm{MoS}_{2}$. 

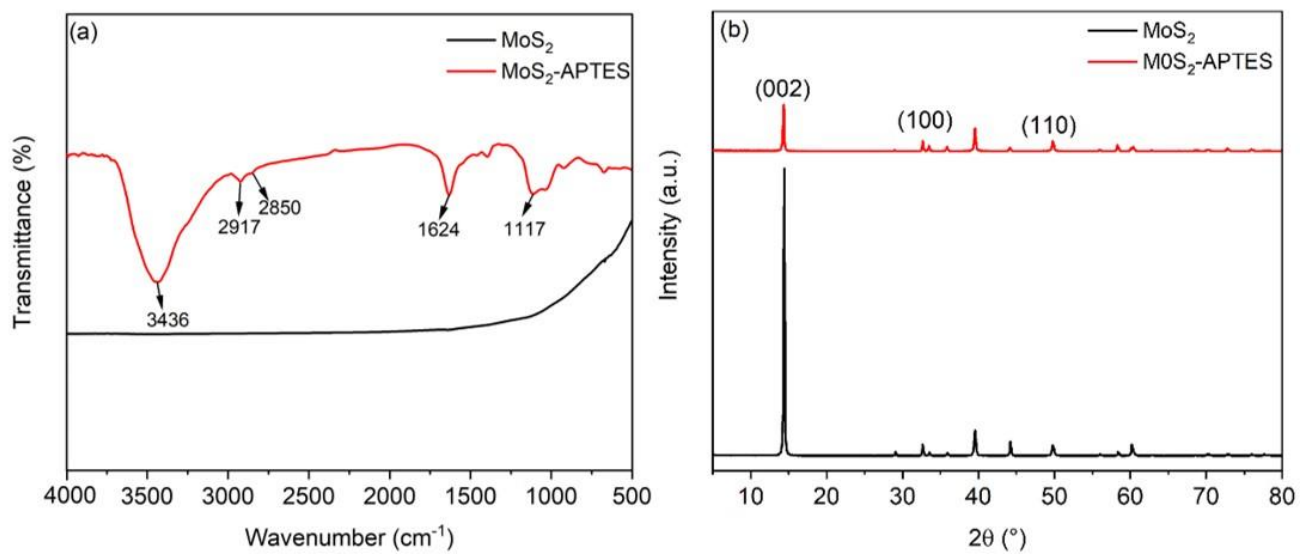

Figure 2. (a) FT-IR spectra and (b) XRD patterns of $\mathrm{MoS}_{2}$, and $\mathrm{MoS}_{2}$-APTES.

In order to explore the morphology of $\mathrm{MoS}_{2}$ and $\mathrm{MoS}_{2}$-APTES, TEM was carried out, and the images are displayed in Figure 3. From Figure 3a, it could be clearly spotted that the average lateral size of $\mathrm{MoS}_{2}$ is close to $500 \mathrm{~nm}$. According to the high magnification TEM image of $\mathrm{MoS}_{2}$-APTES (Figure 3b), a thin film of approximately $5 \mathrm{~nm}$ enveloping the surface of the particle can be seen, which proves that APTES successfully coats on the surface of $\mathrm{MoS}_{2}$ via hydrolysis reaction.

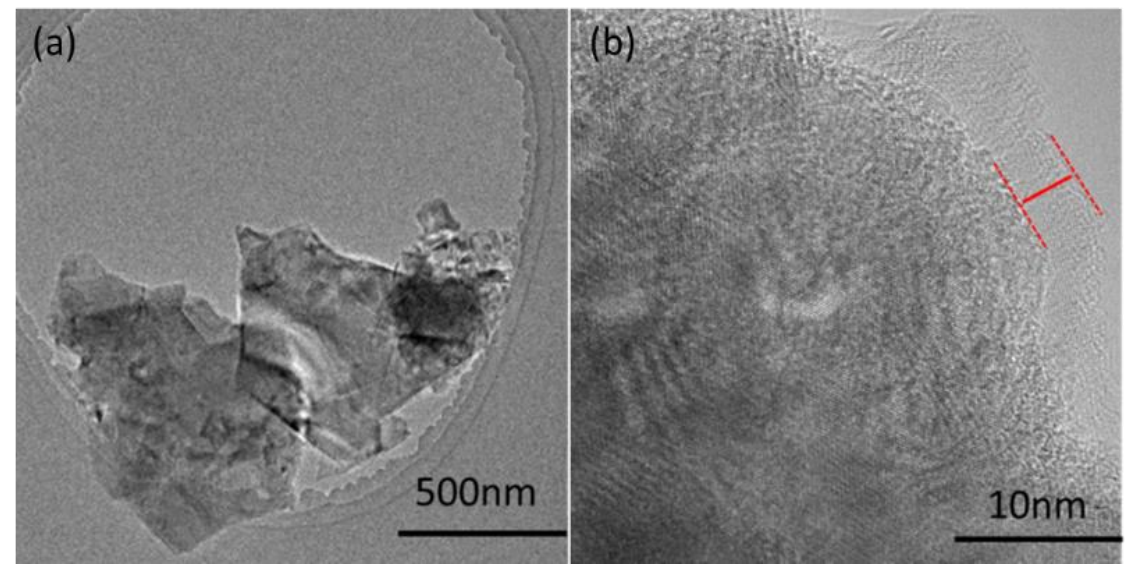

Figure 3. TEM images of (a) $\mathrm{MoS}_{2}$-APTES and (b) high-resolution TEM morphology of $\mathrm{MoS}_{2}$-APTES.

To investigate the chemical element composition of $\mathrm{MoS}_{2}$ modified with APTES, the XPS spectra analysis was performed. The wide-scan spectra of $\mathrm{MoS}_{2}$ and $\mathrm{MoS}_{2}$-APTES are exhibited in Figure 4a. The existences of Mo, S, C and O elements are spotted in both $\mathrm{MoS}_{2}$ and $\mathrm{MoS}_{2}$-APTE samples, and Si and N elements are detected from $\mathrm{MoS}_{2}$-APTES, revealing the successful incorporation of APTES on the surface of $\mathrm{MoS}_{2}$. The peak at $395.6 \mathrm{eV}$ may be overlap between N1s and Mo 3p. The C and O elements of pristine $\mathrm{MoS}_{2}$ may be from the ambient atmosphere. Additionally, the apparent raised C1s and O1s intensity compared with the $\mathrm{MoS}_{2}$ mainly derives from the APTES, which indicates that APTES was also successfully introduced to $\mathrm{MoS}_{2}$. As shown in Figure $4 \mathrm{~b}$, the highresolution spectrum of $\mathrm{C} 1 \mathrm{~s}$ for $\mathrm{MoS}_{2}$-APTES can be deconvoluted into four components situated at 283.60, 284.4, 285.3 and $286.4 \mathrm{eV}$, which correspond to $\mathrm{C}-\mathrm{Si}, \mathrm{C}-\mathrm{C}, \mathrm{C}-\mathrm{N}$ and $\mathrm{C}-\mathrm{O}$ bonds, respectively [32]. In addition, the presence of $\mathrm{C}-\mathrm{N}$ and $\mathrm{C}-\mathrm{C}$ derives from APTES, while $\mathrm{C}-\mathrm{Si}$ is probably created by the dehydration condensation of Silanol [33]. Figure 4c displays the high-resolution $\mathrm{Si} 2 \mathrm{p}$ spectrum which can be split into three peaks. The two peaks at 102.3 and $103.2 \mathrm{eV}$ are observed, which correspond to the $\mathrm{Si}-\mathrm{O}-\mathrm{C}$ and $\mathrm{Si}-\mathrm{O}-\mathrm{Si}$, respectively [11]. The third peak located at $101.8 \mathrm{eV}$ results from the $\mathrm{Si}-\mathrm{C}$ bond. From the high-resolution $\mathrm{Si} 2 \mathrm{p}$ spectrum, it could be concluded that the silane coupling agent 
APTES hydrolyzes during the process of modification, and the produced silanol could undergo dehydration condensation by itself. In addition, the Mo 3d spectrum (Figure 4c) of $\mathrm{MoS}_{2}$-APTES shows five characteristic peaks. The two peaks located at Mo $3 \mathrm{~d}_{5 / 2}(229.2 \mathrm{eV})$

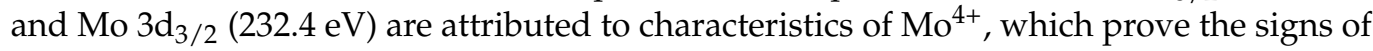
$\mathrm{MoS}_{2}$. In the meantime, the peak appeared at $226.3 \mathrm{eV}$, which is the result of the strong overlap of the S2s and Mo $3 \mathrm{~d}$ region. The peaks at $233.3 \mathrm{eV}$ of Mo $3 \mathrm{~d}_{5 / 2}$ and at $235.7 \mathrm{eV}$ of $\mathrm{Mo} 3 \mathrm{~d}_{3 / 2}$ are characteristic of $\mathrm{Mo}^{6+}$ species, which signify the crosslinking systems between $\mathrm{MoS}_{2}$ and APTES [34]. All of these XPS features reveal the successful modification of APTES on the surface of $\mathrm{MoS}_{2}$.
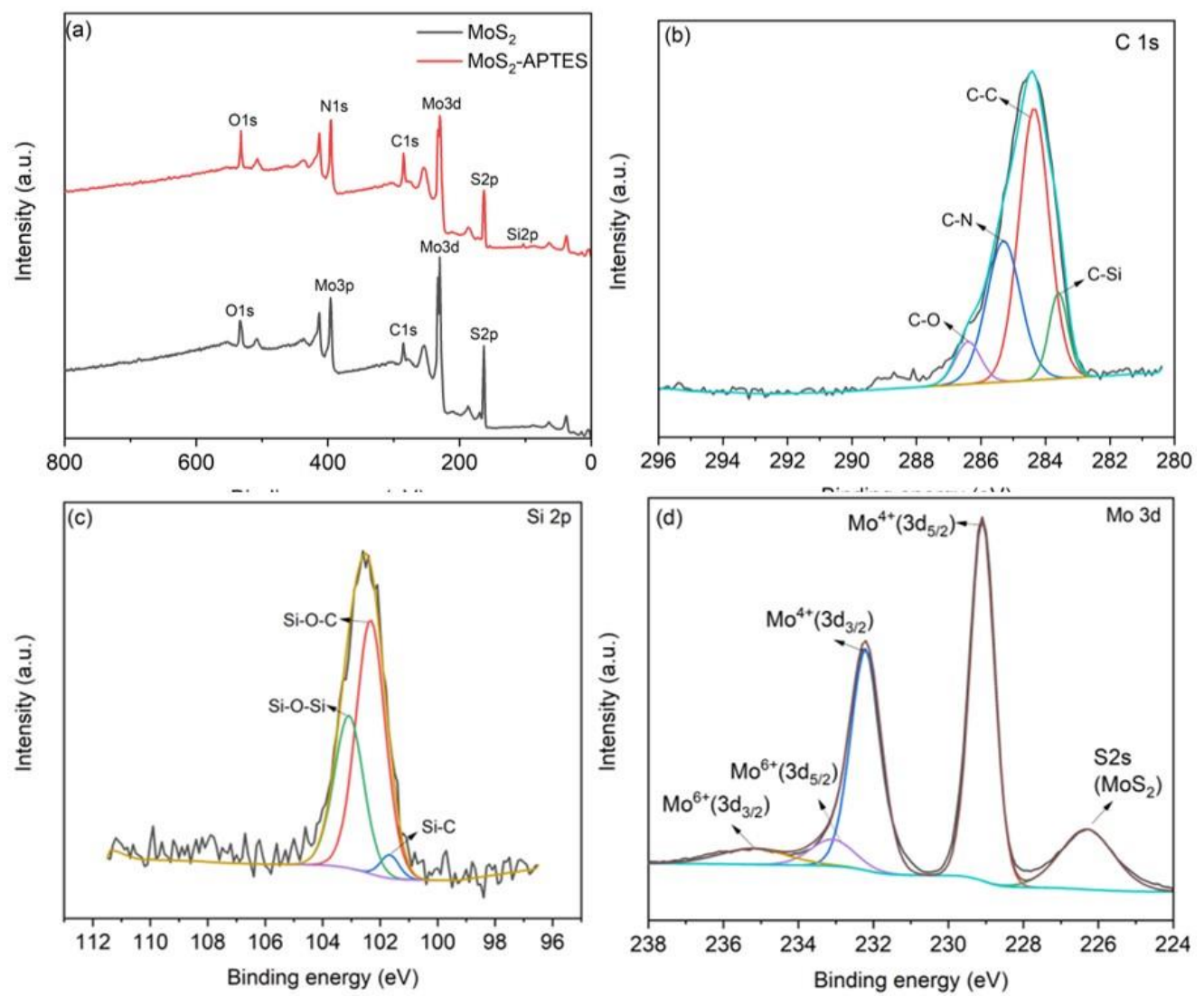

Figure 4. (a) Wide XPS survey of $\mathrm{MoS}_{2}$ and $\mathrm{MoS}_{2}$-APTES and high-resolution spectra in (b) C 1s; (c) Si $2 \mathrm{p}$ and (d) Mo 3d binding energy regions of $\mathrm{MoS}_{2}$-APTES.

The thermal stability of $\mathrm{MoS}_{2}$ and modified $\mathrm{MoS}_{2}$ was studied via TGA from $40{ }^{\circ} \mathrm{C}$ to $800{ }^{\circ} \mathrm{C}$ under $\mathrm{N}_{2}$ atmosphere. The TGA curves of the initial $\mathrm{MoS}_{2}$ and $\mathrm{MoS}_{2}$-APTES hybrids are exhibited in Figure 5. There is no obvious loss of weight during the thermal decomposition procedure of the original $\mathrm{MoS}_{2}$, manifesting its excellent thermally stability in a nitrogen atmosphere [24]. For $\mathrm{MoS}_{2}$-APTES, an enormous amount of decline in weight is attributed to the thermal decomposition of APTES on the surface of $\mathrm{MoS}_{2}$ and the evaporation of the residual water. At the same time, the TGA curves also prove that the amount of APTES in $\mathrm{MoS}_{2}$-APTES hybrids is about $19.4 \mathrm{wt} . \%$. 


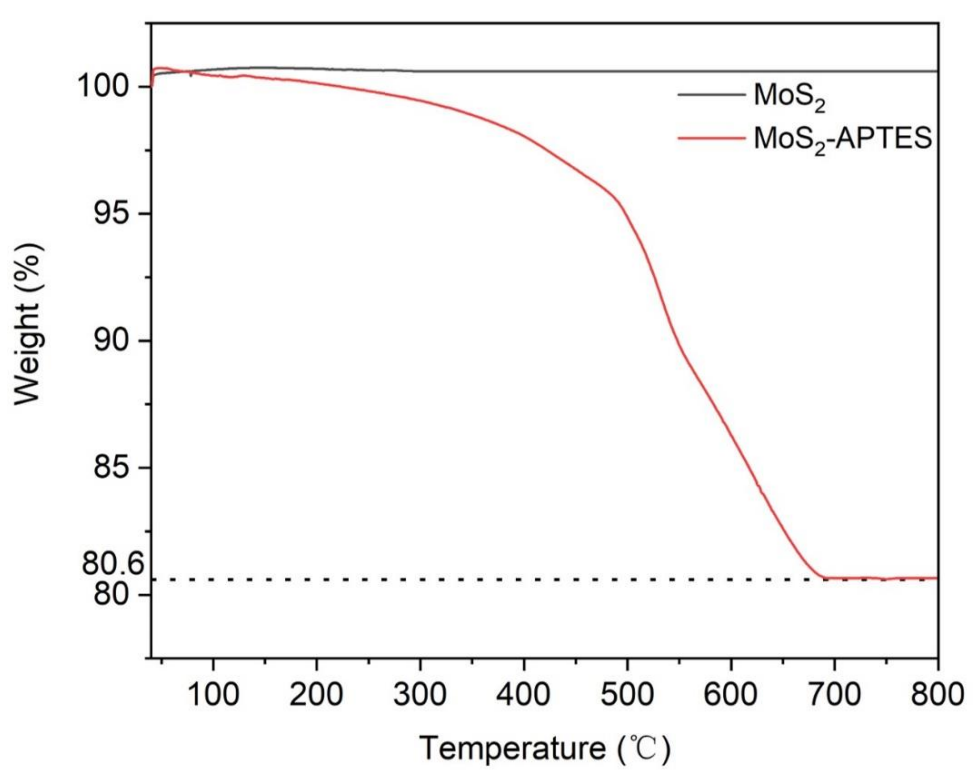

Figure 5. TGA curves of $\mathrm{MoS}_{2}$ and $\mathrm{MoS}_{2}$-APTES.

The fracture surface of different coatings was investigated by SEM and the images are displayed in Figure 6. For the neat WEP coating (Figure 6a), the fracture surface of it is relatively smooth [35]. After adding $\mathrm{MoS}_{2}$ into coating, it could be clearly spotted that the fracture surface of composite coating (Figure 6b) gets tough. However, some serious agglomeration of $\mathrm{MoS}_{2}$ can be observed, because the pristine $\mathrm{MoS}_{2}$ cannot effectively disperse in WEP resin and lacks interfacial bonding between $\mathrm{MoS}_{2}$ and resin [36]. In contrast with these, there is no obvious agglomeration in the $\mathrm{MoS}_{2}$-APTES/WEP composite coating (Figure 6c) and the $\mathrm{MoS}_{2}$ after the modification of APTES is well dispersed in waterborne epoxy resin, which highlights the better compatibility between $\mathrm{MoS}_{2}$-APTES and WEP. This still suggests that the $\mathrm{MoS}_{2}$-APTES/WEP composite coating can provide steel with superior corrosion resistance performance to corrosive media.
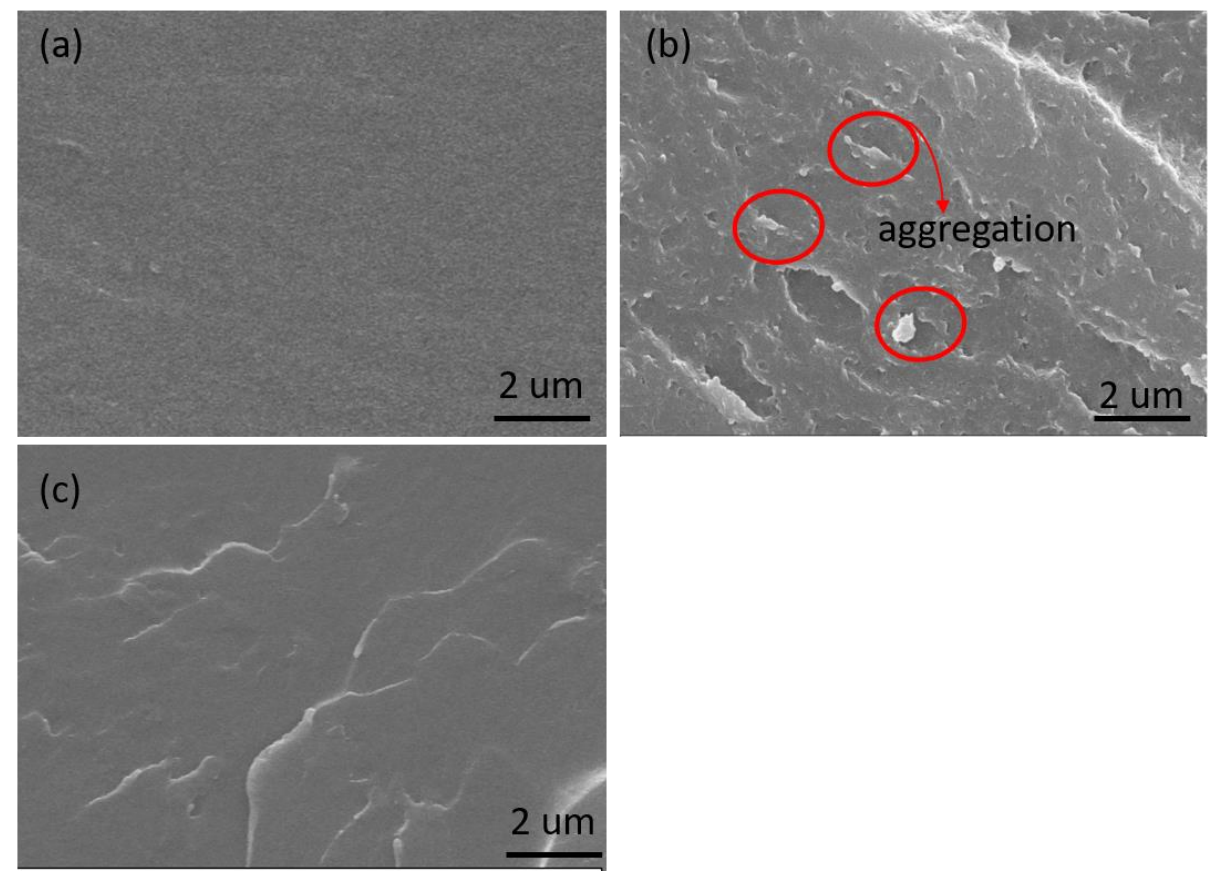

Figure 6. SEM images of the fracture surface of neat waterborne epoxy coating (WEP) (a); $\mathrm{MoS}_{2} / \mathrm{WEP}$ (b); $\mathrm{MoS}_{2}$-APTES/WEP (c). 


\subsection{EIS Test}

EIS was employed to investigate the corrosion resistance performance of the waterborne epoxy coatings at the steady open circuit potentials after an increasing amount of immersion time. The achieved studying data were drawn as Nyquist and Bode diagrams through the immersion time for 5, 15, 30, and 50 days. From Figure 7a,c,e, it could be obviously spotted that with the extending immersion time, the impedance diameters of all the epoxy coatings expose a decline in the Nyquist plots, which means that the anticorrosion property of the coatings is dwindling. As shown in Figure 7a,b, the pure WEP coating only has a semi-arc and one time constant when the immersion time is at 5 days. However, as the time extends to 15 days, the second time constant and two arcs appear, demonstrating that pure WEP coating cannot maintain the decent ability of corrosion resistance for N80 steel. Even though the arcs diameter of the $\mathrm{MoS}_{2} / \mathrm{WEP}$ coating (Figure $7 \mathrm{~b}$ ) was much larger than that of the neat WEP coating at the original immersion in $3.5 \mathrm{wt} . \% \mathrm{NaCl}$ solution (5 days), it dramatically plunges and the second time constant appears similarly when the immersion time merely increases to 15 days. In this situation, the bad dispersibility and compatibility of $\mathrm{MoS}_{2}$ in the WEP coating are the main factors. In obvious contrast to the neat WEP and $\mathrm{MoS}_{2} / \mathrm{WEP}, \mathrm{MoS}_{2}$-APTES/WEP coating (Figure 7e), exhibits the largest impedance arcs after the same immersion time and has only one semi-arcs. Additionally, this compound coating keeps one time constant from 5 to 50 days immersion, which proves that $\mathrm{MoS}_{2}$-APTES coating possesses an outstanding barrier ability and an excellent anticorrosion property.

In general, the lowest impedance moduli $\left(|\mathrm{Z}|_{0.01 \mathrm{~Hz}}\right)$ can be used as an effective method to assess the anticorrosive performance of the coating, which means that the higher $|Z|_{0.01 \mathrm{~Hz}}$ of the coating, the better its protective ability [37]. For Figure $7 \mathrm{~b}$, it is clearly displayed that the neat WEP coating owns a low original value of $|\mathrm{ZI}|_{0.01 \mathrm{~Hz}}$ about $3.5 \times 10^{7} \Omega \cdot \mathrm{cm}^{2}$. Then, the impedance value dives heavily with the increase in the immersion period because of the entering of corrosive medium $\left(\mathrm{O}_{2}, \mathrm{H}_{2} \mathrm{O}, \mathrm{Cl}^{-}\right)$through the coating's micropores or cracks. The variation of the $\mid \mathrm{ZI}_{0.01 \mathrm{~Hz}}$ value of $\mathrm{MoS}_{2} /$ WEP (Figure 7d) coating has a similar tendency to the WEP coating, which is derived from the agglomeration of $\mathrm{MoS}_{2}$ in WEP resin. The $\mathrm{MoS}_{2}$-APTES/WEP coating (Figure 7f) shows the largest $|\mathrm{ZI}|_{0.01 \mathrm{~Hz}}$ value $\left(2.738 \times 10^{9} \Omega \cdot \mathrm{cm}^{2}\right)$. Above all, its value also has $3.647 \times 10^{7} \Omega \cdot \mathrm{cm}^{2}$ even after the immersion time of 50 days in $3.5 \mathrm{wt} . \% \mathrm{NaCl}$ solution, which can be ascribed to the homogeneous dispersion of $\mathrm{MoS}_{2}$-APTES in the WEP matrix and the interactions with them. All of the results prove the addition of $\mathrm{MoS}_{2}$-APTES into WEP coating and can offer carbon steel a long-period anticorrosion protection capacity.

The electrical equivalent circuit of ZSimpWin was used to fit the EIS data and was exhibited in Figure 8. The electrochemical parameters including $\mathrm{CPE}_{\mathrm{C}}$ and $\mathrm{CPE}_{\mathrm{dl}}$ represent the coating capacitance and double-layer capacitance, respectively. $R_{c}$, Rs, and $R_{c t}$ denote the coating resistance, solution resistance (resistance between the specimen coating electrode and saturated calomel electrode), and charge transfer resistance, respectively [38]. All these electrochemical parameters derived from the EIS charts are summed up in Table 1. The $R_{c}$ values of neat WEP, and $\mathrm{MoS}_{2} / \mathrm{WEP}$ decline constantly following the extension of the immersion period. When the immersion time arrives at 15 days, the $\mathrm{R}_{\mathrm{ct}}$ fitted with the second equivalent circuit model appears, demonstrating that the erosive media reach the interface between the coating and steel going through the coating defects and the protective coating loses the ability of corrosion resistance for metal. In contrast, the $\mathrm{MoS}_{2}$-APTES/WEP coating exposes the higher $R_{c}$ value and no $R_{c t}$ value even longing for an immersion time of 50 days, which indicates that the $\mathrm{MoS}_{2}$-APTES/WEP coating is endowed with the superior capability of blocking the penetration of corrosive media. These are another powerful evidence that the addition of $\mathrm{MoS}_{2}$-APTES could enhance the anticorrosion of WEP coating. 

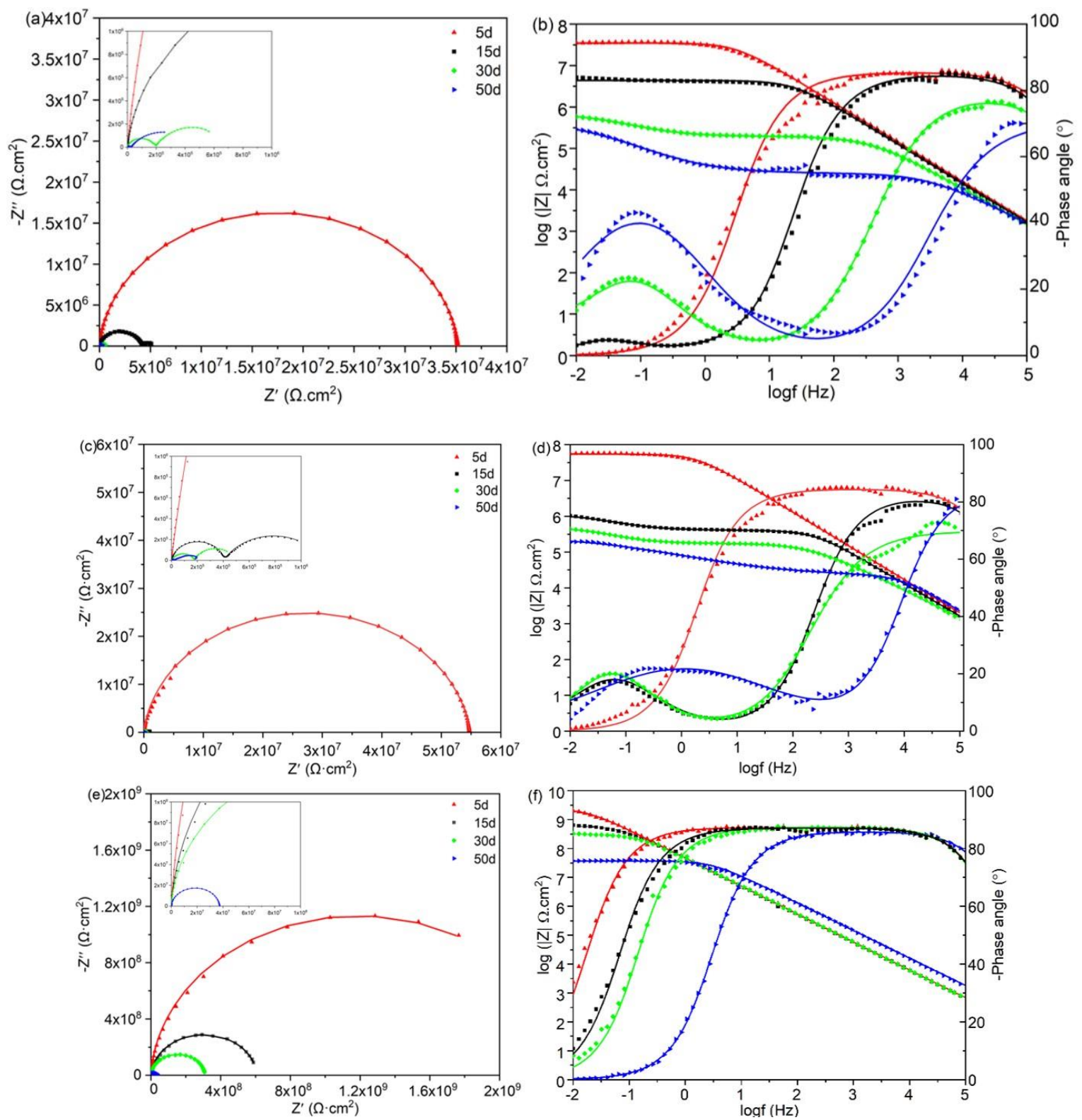

Figure 7. Nyquist and Bode plots of the neat WEP (a,b), $\operatorname{MoS}_{2} / \operatorname{WEP}(\mathbf{c}, \mathbf{d})$ and $\mathrm{MoS}_{2}-\mathrm{APTES} / \mathrm{WEP}(\mathbf{e}, \mathbf{f})$ in a 3.5 wt.\% $\mathrm{NaCl}$ solution.

(a)

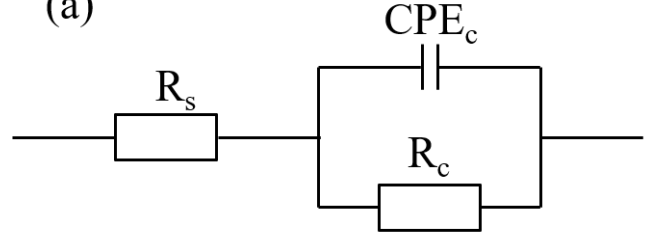

(b)

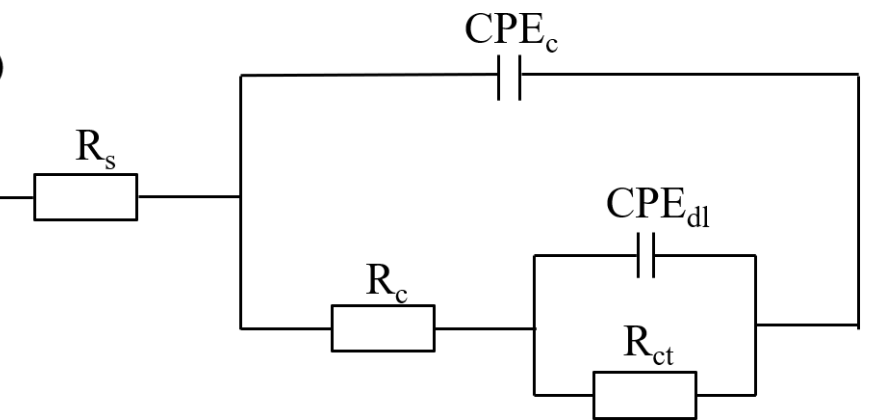

Figure 8. The equivalent circuit models of the first (a) and the second (b). 
Table 1. Electrochemical impedance data of neat and composite waterborne epoxy coatings.

\begin{tabular}{|c|c|c|c|c|c|c|c|}
\hline Sample. & Time & $\mathrm{CPE}_{\mathrm{c}}$ & & $R_{c}\left(\Omega \cdot \mathrm{cm}^{2}\right)$ & $\mathrm{CPE}_{\mathrm{dl}}$ & & $R_{c t}\left(\Omega \cdot \mathrm{cm}^{2}\right)$ \\
\hline & & $\mathrm{Y}_{0}\left(\mathrm{ohm}^{-1} \mathrm{~cm}^{-2} \mathrm{~s}^{\mathrm{n}}\right)$ & $\mathrm{n}_{\text {coat }}$ & & $\mathrm{Y}_{0}\left(\mathrm{ohm}^{-1} \mathrm{~cm}^{-2} \mathrm{~s}^{\mathrm{n}}\right)$ & $\mathrm{n}_{\mathrm{dl}}$ & \\
\hline \multirow[t]{4}{*}{ Epoxy } & $5 \mathrm{~d}$ & $1.736 \times 10^{-9}$ & 0.953 & $3.517 \times 10^{7}$ & - & - & - \\
\hline & $15 \mathrm{~d}$ & $2.043 \times 10^{-8}$ & 0.945 & $4.355 \times 10^{6}$ & $2.145 \times 10^{-6}$ & 0.73 & $1.19 \times 10^{6}$ \\
\hline & $30 \mathrm{~d}$ & $1.4 \times 10^{-8}$ & 0.795 & $1.911 \times 10^{5}$ & $6.552 \times 10^{-6}$ & 0.68 & $5.572 \times 10^{5}$ \\
\hline & $50 \mathrm{~d}$ & $1.369 \times 10^{-5}$ & 0.67 & $4.448 \times 10^{5}$ & $1.779 \times 10^{-5}$ & 0.62 & $2.543 \times 10^{5}$ \\
\hline \multirow[t]{4}{*}{$\mathrm{MoS}_{2}$} & $5 \mathrm{~d}$ & $1.808 \times 10^{-9}$ & 0.94 & $5.476 \times 10^{7}$ & - & - & - \\
\hline & $15 \mathrm{~d}$ & $3.036 \times 10^{-8}$ & 0.92 & $4.05 \times 10^{6}$ & $4.258 \times 10^{-6}$ & 0.78 & $3.688 \times 10^{6}$ \\
\hline & $30 \mathrm{~d}$ & $2.155 \times 10^{-8}$ & 0.78 & $1.763 \times 10^{5}$ & $7.106 \times 10^{-6}$ & 0.73 & $3.225 \times 10^{5}$ \\
\hline & $50 \mathrm{~d}$ & $1.614 \times 10^{-5}$ & 0.663 & $2.272 \times 10^{4}$ & $6.049 \times 10^{-5}$ & 0.64 & $2.929 \times 10^{5}$ \\
\hline \multirow[t]{4}{*}{$\mathrm{MoS}_{2}$-APTES } & $5 \mathrm{~d}$ & $3.59 \times 10^{-10}$ & 0.968 & $2.738 \times 10^{9}$ & - & - & - \\
\hline & $15 \mathrm{~d}$ & $3.757 \times 10^{-9}$ & 0.96 & $6.387 \times 10^{8}$ & - & - & - \\
\hline & $30 \mathrm{~d}$ & $3.565 \times 10^{-9}$ & 0.958 & $3.091 \times 10^{8}$ & - & - & - \\
\hline & $50 \mathrm{~d}$ & $1.63 \times 10^{-9}$ & 0.925 & $3.647 \times 10^{7}$ & - & - & - \\
\hline
\end{tabular}

Potentiodynamic polarization test of the coatings was performed after immersing in $3.5 \mathrm{wt} . \%$ aqueous $\mathrm{NaCl}$ solution for 50 days and polarization diagrams were displayed in Figure 9. Generally speaking, if the protection coating has a higher corrosion potential $\left(\mathrm{E}_{\mathrm{corr}}\right)$ and a lower corrosion current density ( $\left.\mathrm{i}_{\mathrm{corr}}\right)$, it will be endowed with stronger anticorrosion property [39]. According to Figure 9, it can be apparently spotted that the $\mathrm{MoS}_{2}$-APTES/WEP coating shows more positive $\mathrm{E}_{\text {corr }}$ in contrast to $\mathrm{MoS}_{2} / \mathrm{WEP}$ and a neat WEP coating. In addition, the $\mathrm{i}_{\text {corr }}$ of $\mathrm{MoS}_{2}$-APTES/WEP coating is transparently lower than the others. The obtained results can be clarified via two explanations. One is that well distributed $\mathrm{MoS}_{2}$ modified by APTES could prolong the corrosive media's routes to the substrate; another is that amine groups from APTES could lessen flaws in the WEP coating by increasing the crosslink density of the coating. Thus, $\mathrm{MoS}_{2}$-APTES/WEP coating displays the highest anticorrosion result among the three types of coatings.

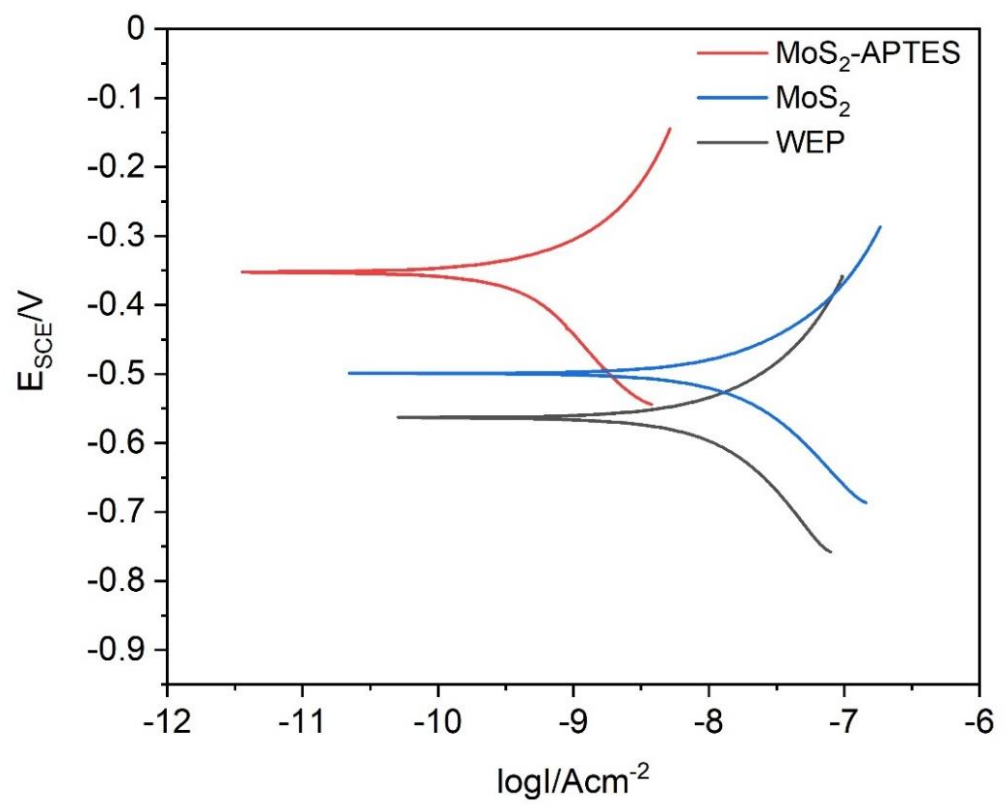

Figure 9. The polarization curves of composite coatings after in $3.5 \mathrm{wt} \% \mathrm{NaCl}$ solution for 50 days.

\subsection{Salt Spray Test}

A salt spray test was employed to further evaluate the long-term anticorrosion property of coatings. The images of coatings, with $4 \mathrm{~cm}$ scratches on their surfaces after being made, put in salt spray of $5 \mathrm{wt} . \% \mathrm{NaCl}$ for 5 and 15 days are displayed in Figure 10. For a neat WEP coating (Figure 10a,d), there are clear bubbles and corrosion products in the 
vicinity of the scratch after the exposure time reaches 5 days, exposing the inferior anticorrosion property of the neat waterborne epoxy coating. As the time extends to 15 days, the corrosion phenomenon increases heavily and much more corrosion products can be easily observed around the scratch, which are caused by more corrosion medium, which reaches the metal surface through coating flaws. After the addition of $\mathrm{MoS}_{2}$ (Figure 10b,e), the $\mathrm{MoS}_{2}$ /WEP coating exhibits a similar corrosion protection result to the neat WEP coating during the salt spray test, because the bad dispersion of $\mathrm{MoS}_{2}$ in WEP and could not act as an effective barrier for the corrosive medium. In contrast to the neat WEP coating and $\mathrm{MoS}_{2}$ /WEP coating, there are less corrosion products on the surface of $\mathrm{MoS}_{2}$-APTES/WEP composite coating (Figure 10c,f) even after 15 days of exposure time. The obtained results above prove that the $\mathrm{MoS}_{2}$-APTES/WEP coating has superior and long-term corrosion protection because of the physical barrier of $\mathrm{MoS}_{2}$-APTES.
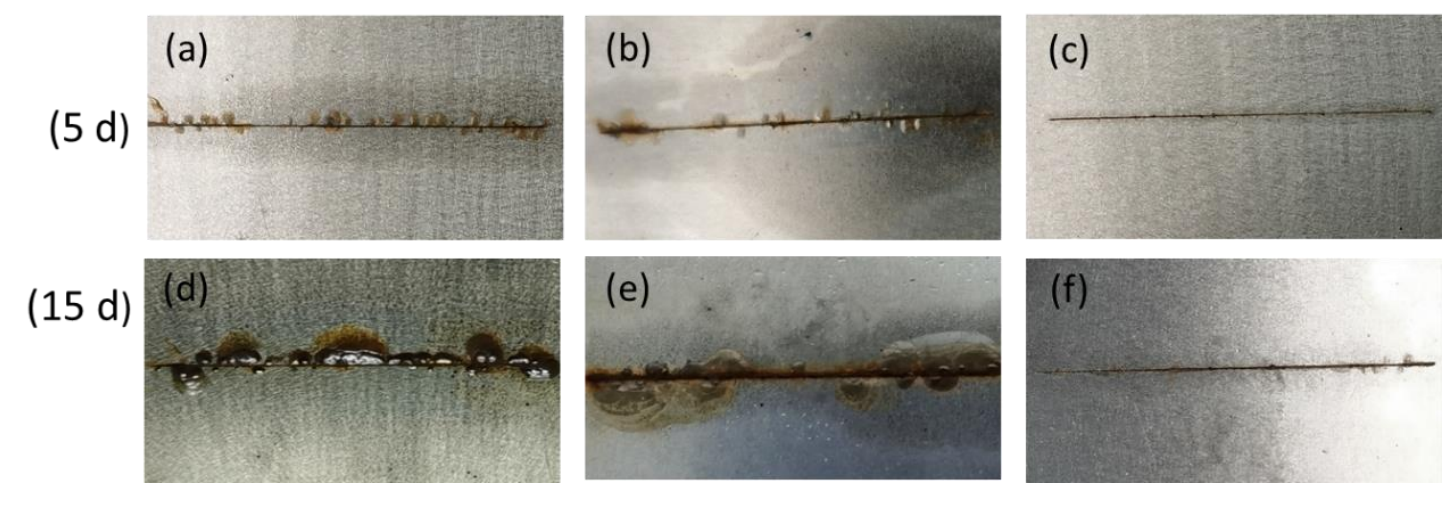

Figure 10. The visual picture of the neat $\operatorname{WEP}(\mathbf{a}, \mathbf{d}), \operatorname{MoS}_{2} / \operatorname{WEP}(\mathbf{b}, \mathbf{e})$ and $\operatorname{MoS}_{2}$-APTES/WEP $(\mathbf{c}, \mathbf{f})$ samples after 5 and 15 days.

\subsection{The Corrosion Protection Mechanism}

It can be concluded that the $\mathrm{MoS}_{2}$-APTES/WEP coating is endowed with a longterm and superior corrosion protection property to M80 steel, considering the results from the relevant EIS and salt spray test above. The corrosion protection mechanism of the $\mathrm{MoS}_{2}$-APTES/WEP coating is shown in Figure 11. In terms of neat WEP coating (Figure 11a), corrosive electrolytes such as $\mathrm{H}_{2} \mathrm{O}, \mathrm{O}_{2}, \mathrm{Cl}^{-}$can arrive at the interface between the coating and steel through diffusion channels, leading to metal corrosion [40]. Though $\mathrm{MoS}_{2}$ is added into the WEP coating, the $\mathrm{MoS}_{2}$ /WEP coating (Figure 11b) cannot function effectively as a barrier impact on corrosive medium, because of its inferior dispersion in WEP. From Figure 11c, $\mathrm{MoS}_{2}$-APTES exposes homogeneous dispersion in WEP coating, giving rise to much more better barrier performance and more superior corrosion resistance properties, which is ascribed to the excellent compatibility of $\mathrm{MoS}_{2}$ and WEP. Moreover, amine-end groups of APTES could improve the crosslinking density between $\mathrm{MoS}_{2}$-APTES and WEP matrix.

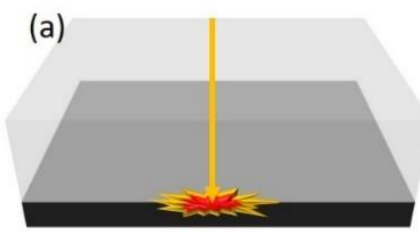

Permeation pathways

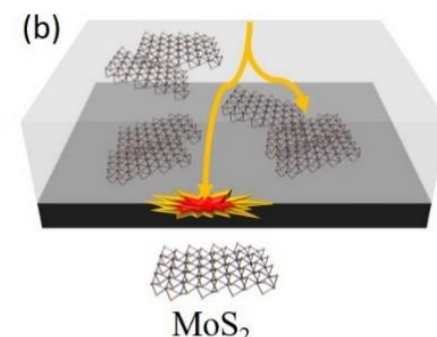

$\mathrm{MoS}_{2}$

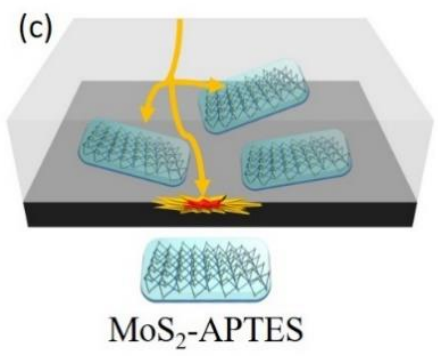

Figure 11. Schematic illustration of a corrosion protection mechanism for neat WEP (a), $\mathrm{MoS}_{2} / \mathrm{WEP}(\mathbf{b})$ and $\mathrm{MoS}_{2}-$ APTES/WEP (c) coating. 


\section{Conclusions}

In this work: a simple form of the anticorrosive addition $\mathrm{MoS}_{2}$-APTES was developed, which linked the virtues between $\mathrm{MoS}_{2}$ and APTES. Then, an $\mathrm{MoS}_{2}$-APTES hybrid, serving as a pigment, was added into the waterborne epoxy, which shows an apparently enhanced anticorrosion performance which effectively protects the N80 steel matrix. The successful modification of APTES on $\mathrm{MoS}_{2}$ is proved by FT-IR, XRD, TEM, XPS, and TGA. Moreover, the results of the SEM, EIS, potentiodynamic polarization and salt spray test reveal a long-term corrosion resistance of $\mathrm{MoS}_{2}$-APTES/WEP. The extraordinary performance of corrosion resistance is derived from the good dispersity of $\mathrm{MoS}_{2}$-APTES which can prolong the diffusion path of corrosive media going through the coating and APTES can strengthen the crosslinking density of waterborne epoxy resin.

Author Contributions: Conceptualization, Y.L. and S.Z.; methodology, Y.L.; software, P.X.; validation, Z.L., J.C. and F.Z.; formal analysis, C.Z.; investigation, Y.L.; resources, Y.H.; data curation, H.L.; writing-original draft preparation, Y.L.; writing-review and editing, C.C.; visualization, C.Z.; supervision, S.Z.; project administration, Z.L.; funding acquisition, Y.H. All authors have read and agreed to the published version of the manuscript.

Funding: Open Fund (PLN161, PLN201806) of State Key Laboratory of Oil and Gas Reservoir Geology and Exploitation (Southwest Petroleum University), the Research Program of Science and Technology Department of Sichuan Province (2018JY0302, 18YYJC0018) and the National Natural Science Foundation of China (51774245).

Data Availability Statement: No new data were created or analyzed in this study. Data sharing is not applicable to this article.

Conflicts of Interest: The authors declare no conflict of interest.

\section{References}

1. Ding, J.; Zhao, H.; Shao, Z.; Yu, H. Bioinspired Smart Anticorrosive Coatings with an Emergency-Response Closing Function. ACS Appl. Mater. Interfaces 2019, 11, 42646-42653. [CrossRef] [PubMed]

2. Li, Y.; Yang, Z.; Qiu, H.; Dai, Y.; Zheng, Q.; Li, J.; Yang, J. Self-aligned graphene as anticorrosive barrier in waterborne polyurethane composite coatings. J. Mater. Chem. A 2014, 2, 14139-14145. [CrossRef]

3. Ramezanzadeh, B.; Haeri, Z. A facile route of making silica nanoparticles-covered graphene oxide nanohybrids (SiO2-GO); Fabrication of $\mathrm{SiO} 2-\mathrm{GO}$ /epoxy composite coating with superior barrier and corrosion protection performance. Chem. Eng. J. 2016, 303, 511-528. [CrossRef]

4. Ramezanzadeh, B.; Niroumandrad, S.; Ahmadi, A.; Mahdavian, M.; Moghadam, M.M. Enhancement of barrier and corrosion protection performance of an epoxy coating through wet transfer of amino functionalized graphene oxide. Corros. Sci. 2016, 103, 283-304. [CrossRef]

5. Cui, M.J.; Ren, S.M.; Zhao, H.C.; Xue, Q.J.; Wang, L.P. Polydopamine coated graphene oxide for anticorrosive rein-forcement of water-borne epoxy coating. Chem. Eng. J. 2018, 335, 255-266. [CrossRef]

6. Cui, M.J.; Ren, S.M.; Chen, J.; Liu, S.; Zhang, G.G.; Zhao, H.C.; Wang, L.P.; Xue, Q.J. Anticorrosive performance of wa-terborne epoxy coatings containing water-dispersible hexagonal boron nitride (h-BN) nanosheets. Appl. Surf. Sci. 2017, 397, 77-86. [CrossRef]

7. Ding, J.; Rahman, O.U.; Peng, W.; Dou, H.; Yu, H. A novel hydroxyl epoxy phosphate monomer enhancing the anticorrosive performance of waterborne Graphene/Epoxy coatings. Appl. Surf. Sci. 2018, 427, 981-991. [CrossRef]

8. Chen, C.L.; Xiao, G.Q.; He, Y.; Zhong, F.; Li, H.J.; Wu, Y.Q.; Chen, J.Y. Bio-inspired superior barrier self-healing coating: Selfassemble of graphene oxide and polydopamine-coated halloysite nanotubes for enhancing corrosion resistance of waterborne epoxy coating. Prog. Org. Coat. 2020, 139, 105462. [CrossRef]

9. Wu, Y.; He, Y.; Zhou, T.; Chen, C.; Zhong, F.; Xia, Y.; Xie, P.; Zhang, C. Synergistic functionalization of h-BN by mechanical exfoliation and PEI chemical modification for enhancing the corrosion resistance of waterborne epoxy coating. Prog. Org. Coat. 2020, 142, 105541. [CrossRef]

10. Zhong, F.; He, Y.; Wang, P.Q.; Chen, C.L.; Yu, H.; Li, H.J.; Chen, J.Y. Graphene/V2O5@polyaniline ternary composites enable waterborne epoxy coating with robust corrosion resistance. React. Funct. Polym. 2020, 151, 104567. [CrossRef]

11. Xia, Y.; He, Y.; Chen, C.; Wu, Y.; Zhong, F.; Chend, J. Co-modification of polydopamine and KH560 on g-C3N4 nanosheets for enhancing the corrosion protection property of waterborne epoxy coating. React. Funct. Polym. 2020, 146, 104405. [CrossRef]

12. Zhong, F.; He, Y.; Wang, P.; Chen, C.; Lin, Y.; Wu, Y.; Chen, J. Self-assembled graphene oxide-graphene hybrids for enhancing the corrosion resistance of waterborne epoxy coating. Appl. Surf. Sci. 2019, 488, 801-812. [CrossRef] 
13. Chang, C.-H.; Huang, T.-C.; Peng, C.-W.; Yeh, T.-C.; Lu, H.-I.; Hung, W.-I.; Weng, C.-J.; Yang, T.-I.; Yeh, J.-M. Novel anticorrosion coatings prepared from polyaniline/graphene composites. Carbon 2012, 50, 5044-5051. [CrossRef]

14. Zhang, C.L.; He, Y.; Li, F.; Di, H.H.; Zhang, L.; Zhan, Y.Q. h-BN decorated with Fe3O4 nanoparticles through mus-sel-inspired chemistry of dopamine for reinforcing anticorrosion performance of epoxy coatings. J. Alloys Compd. 2016, 685, 743-751. [CrossRef]

15. Shi, H.; Liu, W.; Liu, C.; Yang, M.; Xie, Y.; Wang, S.; Zhang, F.; Liang, L.; Pi, K. Polyethylenimine-assisted exfoliation of h-BN in aqueous media for anticorrosive reinforcement of waterborne epoxy coating. Prog. Org. Coat. 2020, 142, 105591. [CrossRef]

16. Li, Y.; Wang, H.; Xie, L.; Liang, Y.; Hong, G.; Dai, H. MoS2Nanoparticles Grown on Graphene: An Advanced Catalyst for the Hydrogen Evolution Reaction. J. Am. Chem. Soc. 2011, 133, 7296-7299. [CrossRef]

17. Xiang, Q.; Yu, J.; Jaroniec, M. Synergetic Effect of MoS2and Graphene as Cocatalysts for Enhanced Photocatalytic H2Production Activity of TiO2Nanoparticles. J. Am. Chem. Soc. 2012, 134, 6575-6578. [CrossRef]

18. Lopez-Sanchez, O.; Lembke, D.; Kayci, M.; Radenovic, A.; Kis, A. Ultrasensitive photodetectors based on monolayer MoS2. Nat. Nanotechnol. 2013, 8, 497-501. [CrossRef]

19. Lee, Y.-H.; Zhang, X.Q.; Zhang, W.; Chang, M.-T.; Lin, C.-T.; Chang, K.-D.; Yu, Y.-C.; Wang, J.T.-W.; Chang, C.-S.; Li, L.-J.; et al. Synthesis of Large-Area MoS2Atomic Layers with Chemical Vapor Deposition. Adv. Mater. 2012, 24, 2320-2325. [CrossRef]

20. Zhao, X.; Zhang, B.; Jin, Z.; Chen, C.; Zhu, Q.; Hou, B. Epoxy coating modified by 2D MoS2/SDBS: Fabrication, anticorrosion behaviour and inhibition mechanism. RSC Adv. 2016, 6, 97512-97522. [CrossRef]

21. Xia, Z.; Liu, G.; Dong, Y.; Zhang, Y. Anticorrosive epoxy coatings based on polydopamine modified molybdenum disulfide. Prog. Org. Coat. 2019, 133, 154-160. [CrossRef]

22. Jing, Y.; Wang, P.; Yang, Q.; He, Y.; Bai, Y. Molybdenum disulfide with poly(dopamine) and epoxy groups as an efficiently anticorrosive reinforcers in epoxy coating. Synth. Met. 2020, 259, 116249. [CrossRef]

23. Ding, J.; Zhao, H.; Zhao, X.; Xu, B.; Yu, H. How semiconductor transition metal dichalcogenides replaced graphene for enhancing anticorrosion. J. Mater. Chem. A 2019, 7, 13511-13521. [CrossRef]

24. Zhou, K.; Liu, C.; Gao, R. Polyaniline: A novel bridge to reduce the fire hazards of epoxy composites. Compos. Part A Appl. Sci. Manuf. 2018, 112, 432-443. [CrossRef]

25. Wang, D.; Song, L.; Zhou, K.Q.; Yu, X.J.; Hu, Y.; Wang, J. Anomalous nano-barrier effects of ultrathin molybdenum di-sulfide nanosheets for improving the flame retardance of polymer nanocomposites. J. Mater. Chem. A 2015, 3, 14307-14317. [CrossRef]

26. Zhou, K.; Jiang, S.; Bao, C.; Song, L.; Wang, B.; Tang, G.; Hu, Y.; Gui, Z. Preparation of poly(vinyl alcohol) nanocomposites with molybdenum disulfide (MoS2): Structural characteristics and markedly enhanced properties. RSC Adv. 2012, 2, 11695-11703. [CrossRef]

27. Pourhashem, S.; Rashidi, A.; Vaezi, M.R.; Bagherzadeh, M.R. Excellent corrosion protection performance of epoxy composite coatings filled with amino-silane functionalized graphene oxide. Surf. Coat. Technol. 2017, 317, 1-9. [CrossRef]

28. Pourhashem, S.; Vaezi, M.R.; Rashidi, A.; Bagherzadeh, M.R. Distinctive roles of silane coupling agents on the corrosion inhibition performance of graphene oxide in epoxy coatings. Prog. Org. Coat. 2017, 111, 47-56. [CrossRef]

29. Zhou, K.; Gao, R.; Qian, X. Self-assembly of exfoliated molybdenum disulfide (MoS 2 ) nanosheets and layered double hydroxide (LDH): Towards reducing fire hazards of epoxy. J. Hazard. Mater. 2017, 338, 343-355. [CrossRef]

30. Asadi, N.; Naderi, R.; Mahdavian, M. Doping of zinc cations in chemically modified halloysite nanotubes to improve protection function of an epoxy ester coating. Corros. Sci. 2019, 151, 69-80. [CrossRef]

31. Karekar, S.E.; Pinjari, D.V. Sonochemical synthesis and characterization of molybdenum sulphide nanoparticles: Effect of calcination temperature. Chem. Eng. Process. Process. Intensif. 2017, 120, 268-275. [CrossRef]

32. Knorr, D.B.; Tran, N.T.; Gaskell, K.J.; Orlicki, J.A.; Woicik, J.C.; Jaye, C.; Fischer, D.A.; Lenhart, J.L. Synthesis and Char-acterization of Aminopropyltriethoxysilane-Polydopamine Coatings. Langmuir 2016, 32, 4370-4381. [CrossRef] [PubMed]

33. Xu, G.M.; Xu, G.N.; Chang, W.G.; Wang, H.Y. Fabrication of microsphere-like nano-MoO2 modified with silane coupling agent KH550. Mater. Res. Express. 2019, 6. [CrossRef]

34. Tanhaei, M.; Ren, Y.; Yang, M.; Bussolotti, F.; Cheng, J.J.W.; Pan, J.; Chiam, S.Y. Direct control of defects in molybdenum oxide and understanding their high $\mathrm{CO}_{2}$ sorption performance. J. Mater. Chem. A 2020, 8, 12576-12585. [CrossRef]

35. Chen, C.; He, Y.; Xiao, G.; Zhong, F.; Xia, Y.; Wu, Y. Graphic C3N4-assisted dispersion of graphene to improve the corrosion resistance of waterborne epoxy coating. Prog. Org. Coat. 2020, 139, 105448. [CrossRef]

36. Hong, M.-S.; Park, Y.; Kim, J.-G.; Kim, K. Effect of Incorporating MoS2 in Organic Coatings on the Corrosion Resistance of 316L Stainless Steel in a 3.5\% NaCl Solution. Coatings 2019, 9, 45. [CrossRef]

37. Zuo, S.X.; Chen, Y.; Liu, W.J.; Yao, C.; Li, Y.R.; Ma, J.Q.; Kong, Y.; Mao, H.H.; Li, Z.Y.; Fu, Y.S. Polyaniline/g-C3N4 com-posites as novel media for anticorrosion coatings. J Coat. Technol. Res. 2017, 14, 1307-1314. [CrossRef]

38. Ramezanzadeh, B.; Moghadam, M.H.M.; Shohani, N.; Mandavian, M. Effects of highly crystalline and conductive polyaniline/graphene oxide composites on the corrosion protection performance of a zinc-rich epoxy coating. Chem. Eng. J. 2017, 320, 363-375. [CrossRef]

39. Zheng, H.; Shao, Y.; Wang, Y.; Meng, G.; Liu, B. Reinforcing the corrosion protection property of epoxy coating by using graphene oxide-poly(urea-formaldehyde) composites. Corros. Sci. 2017, 123, 267-277. [CrossRef]

40. Wang, H.H.; Qin, S.D.; Yang, X.F.; Fei, G.Q.; Tian, M.; Shao, Y.M.; Zhu, K. A waterborne uniform gra-phene-poly(urethane-acrylate) complex with enhanced anticorrosive properties enabled by ionic interaction. Chem. Eng. J. 2018, 351, 939-951. [CrossRef] 